\title{
Current training on the basics of robotic surgery in the Netherlands: Time for a multidisciplinary approach?
}

\author{
Willem Brinkman ${ }^{1}$ - Isabel de Angst ${ }^{1} \cdot$ Henk Schreuder ${ }^{2} \cdot$ Barbara Schout $^{3}$. \\ Werner Draaisma $^{4} \cdot$ Lisanne Verweij $^{5} \cdot$ Ad Hendrikx $^{6} \cdot$ Henk van der Poel ${ }^{7}$
}

Received: 27 October 2015/Accepted: 3 May 2016/Published online: 18 May 2016

(c) The Author(s) 2016. This article is published with open access at Springerlink.com

\begin{abstract}
Introduction The following research questions were answered: (1) What are the training pathways followed by the current robot professionals? (2) Are there any differences between the surgical specialties in robot training and robot use? (3) What is their opinion about multidisciplinary basic skills training?

Methods An online questionnaire was sent to 91 robot professionals in The Netherlands. The questionnaire contained 21 multiple-choice questions focusing on demographics, received robot training, and their opinion on basic skills training in robotic surgery.
\end{abstract}

Electronic supplementary material The online version of this article (doi:10.1007/s00464-016-4970-2) contains supplementary material, which is available to authorized users.

Willem Brinkman

wmbrinkman@gmail.com

1 Department of Urology, Erasmus MC, Rotterdam, The Netherlands

2 UMC Utrecht Cancer Center, Department of Gynaecologic Oncology, University Medical Centre Utrecht, Utrecht, The Netherlands

3 Department of Urology, Alrijne Hospital, Leiden, The Netherlands

4 Department of Surgery, Meander Medical Centre, Amersfoort, The Netherlands

5 The Netherlands Institute of Health Services Research (NIVEL), Utrecht, The Netherlands

6 Department of Urology, Catharina Hospital, Eindhoven, The Netherlands

7 Department of Urology, Netherlands Cancer Institute, Amsterdam, The Netherlands
Results The response rate was $62 \%(n=56): 13$ general surgeons, 16 gynecologists, and 27 urologists. The urologists performed significantly more robotic procedures than surgeons and gynecologists. The kind of training of all professionals varied from a training program by Intuitive Surgical, master-apprenticeship with or without duo console, fellowship, and self-designed training programs. The training did neither differ significantly among the different specialties nor the year of starting robotic surgery. Majority of respondents favor an obliged training program including an examination for the basics of robot skills training.

Conclusion Training of the current robot professionals is mostly dependent on local circumstances and the manufacturer of the robot system. Training is independent of the year of start with robotic surgery and speciality. To guarantee the quality of future training of residents and fellows in robot-assisted surgery, clear training goals should be formulated and implemented. Since this study shows that current training of different specialities does not differ, training in robotic surgery could be started by a multidisciplinary basic skills training and assessment.

Keywords Training · Robot · Surgery · Urology · Gynecology $\cdot$ Simulation

Currently, an increasing number of professionals across several specialties are using robot-assisted laparoscopy to perform surgery [1-3]. The transition from traditional open and laparoscopic surgery to this advanced technology makes procedures different and involves lack of haptic feedback, remote surgical control, and stereoscopic vision compared to laparoscopic surgery or open surgery. The pioneers in robot-assisted laparoscopy encountered new difficulties: master-apprenticeship learning was impossible 
due to the lack of experienced colleagues. Also in robotic surgery, if a supervisor is available, only verbal guidance instead of hands-on assistance can be given, due to the single console, which allows for only one operator. Consequently, simulation training was advocated from the start of robot-assisted laparoscopy. The manufacturer of the da Vinci Surgical System, currently the only approved system for robot-assisted laparoscopy, provided a mandatory startup training program for all new customers. Previous studies report the effectiveness of various simulation-based training tasks for robot surgery, which will shorten the learning curve and improve the technical and nontechnical difficulties of robot-assisted laparoscopy $[4,5]$.

While the first generation of professionals had to learn robot-assisted laparoscopy without a supervisor, there is now a shift to a new generation that has the possible advantage of a supervisor in their hospital. Nevertheless, since these new users are not new customers, the training program provided by the manufacturer is not mandatory. Therefore, a gap now occurs for residents or fellows, who have not received basic training in robot-assisted surgery. This possible gap is equal for the different specialties using the robot. To prevent training by doing surgery directly on patients, and since basic robot training could be equal for the different specialties such as general surgery, gynecology, and urology, a multidisciplinary basic robotic skills training could be a feasible and effective training method. Before such a program can be developed, it is important to first investigate the training pathways of the current robot users, differences between specialities and the opinions of users about multidisciplinary basic skills training.

In this study, we aim to answer the following research questions: (1) What are the training pathways followed by the current robot surgeons? (2) Are there any differences between the specialties in robot training and robot use? (3) What is their opinion about multidisciplinary basic skills training?

\section{Method and materials}

For this nationwide, multidisciplinary study, we developed a specific questionnaire in order to answer our research questions. The questionnaire was online based using Surveymonkey ${ }^{\circledR}$ software. The questionnaire was sent to all known surgical robot users in The Netherlands, identified by their national society. In total, 91 medical specialists were invited to participate in this study. After the initial invitation, one reminder was sent.

\section{Questionnaire}

The questionnaire contained 21 multiple-choice questions. In the first part, demographic variables such as the year of start with robot-assisted laparoscopy and the number of procedures were investigated. In the second part, questions specifically focused on robot training, e.g., what kind of training they had received, how many hours they had spent on training, and how many procedures they had performed under supervision. The final part of the questionnaire focused on their opinion on a basic skills training in robotic surgery.

\section{Data analysis}

Statistical Package for the Social Sciences (SPSS) version 21 was used for the analyses. To analyze the differences between the specialties, we used cross tabs with Chi-square test and correlation was calculated with the Spearman's rho. The alpha level was set at 0.05 .

\section{Results}

Of 91 invited specialists, 56 completed the questionnaire resulting in an overall response rate of $62 \%$ (surgeons $65 \%$, gynecologists $59 \%$, urologists $61 \%$ ). One respondent did not complete the questionnaire and was excluded from the study. Of all included participants, 13 were general surgeons, 16 were gynecologists, and 27 were urologists. The median age was 48 years (range 36-61). Of the respondents, 50 were male and 6 were female.

All respondents had previous laparoscopic experience prior to the start of robotic surgery except for one. The year the specialists had begun performing robotic surgery varied from the year 2000-2014 (surgeons 2000-2014, gynecologist 2006-2013, and urologist 2002-2014) and did not differ significantly between the specialties. According to this questionnaire, urologists performed significantly more robotic procedures than surgeons and gynecologists, and

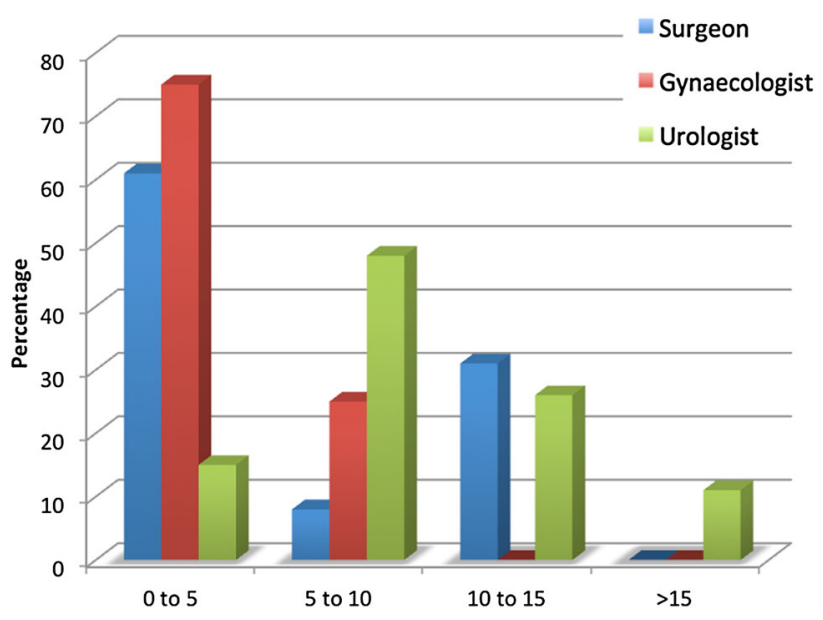

Fig. 1 Number of procedures per month (in percentage) 
surgeons performed more procedures than gynecologists. (Figure 1) $($ Chi-square test, $p$ value $=0.001)$.

\section{Training experience}

All participants had previous laparoscopic experience prior to the start of robotic surgery except for one. The sorts of training varied from a training program by Intuitive Surgical, master-apprenticeship with or without duo console, fellowship, and self-designed training programs (Fig. 2). The hours spent on the different sorts of training are shown in Fig. 3. The training did neither differ significantly among the different specialties nor the year of start.

According to the questionnaire, the surgeons were supervised during a median of $5(0-55)$, the gynecologists during a median of 6 (0-55), and urologists during a median of 10 procedures (2-75). This difference between the different specialties was not significant. Only $10.7 \%$ of the participants used a duo console for supervision. Again, no significant differences between specialties existed (Pearson Chi-square test: 6.247; $p$ value $=0.620$ ). The number of supervised procedures was correlated neither to year of start with robot-assisted laparoscopy nor to age in years (Spearman's rho $=-0.13 ;-0.22$ ).

As for teaching robotic surgical skills, more than half of the respondents $(71.6 \%)$ do so to colleagues, residents, and fellows (Fig. 4). This did not differ significantly among the surgical specialties (Pearson Chi-square test: $3.174 ; p$ value $=$ 0.205 ). Of the teaching respondents, $32.5 \%$ claimed to be a proctor for Intuitive Surgical. The robotic skills education varied from master-apprenticeship with or without duo console to a structured training program created by Intuitive Surgical and a self-designed training curriculum (Fig. 5).

\section{Opinion on multidisciplinary basic skills training}

The respondents were asked whether they agreed on setting a structured and obliged training program for basic robotic

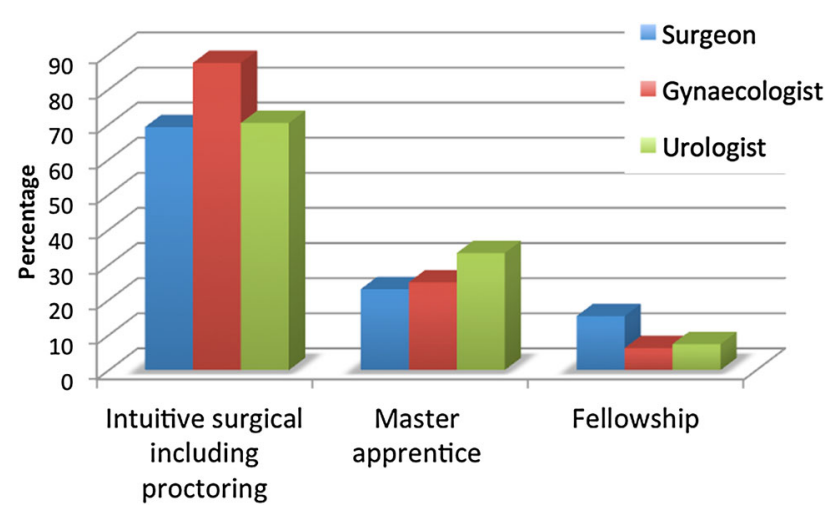

Fig. 2 Type of training experienced skills: $65 \%$ agreed, $25 \%$ disagreed, and $10 \%$ had no opinion. To the question, do you think an exam or test should be installed before starting robot-assisted surgery, $68 \%$ of the respondents agreed, $19 \%$ disagreed, and $12 \%$ had no opinion (Figure 6).

\section{Discussion}

With this study, we aimed to answer three research questions. The first two questions were: What are the training pathways followed by the current robot surgeons? and Are there differences between the specialties in robot training and robot use? The results of the questionnaire demonstrate that urologists use the robot significantly more often than the surgeons and gynecologists. This is not surprising since we know that the da Vinci robot has especially landed in urology [6]. The results of this questionnaire revealed a wide variation of training programs. The kind of training varied from a structured training program by Intuitive Surgical, master-apprenticeship with or without duo console, fellowship, and self-designed training programs. The number of hours differed among individuals, but did not show significant differences between the different specialties. Also, the year of start with robotic surgery did not significantly influence the type or amount of training. Although one should expect that training and especially supervision would increase over the years with an increasing availability of supervisors, our data do not show this. This is in line with previous research among European urologists [7]. Possibly, the heterogeneity among robot starters was too large to show differences. For instance, some 'early' starters were already the second operator in their hospital with an experienced colleague. Also, some 'late' starters were the first in their hospital without a more experienced colleague to teach them.

Remarkable is that the majority of respondents spent less than $5 \mathrm{~h}$ on basic skills training. Recent literature shows an average of $10 \mathrm{~h}$ of skills training is needed to reach proficiency in basic robotic skills [8]. Most likely, the basic skills training of the professionals was time based instead of criterion based. Recent literature data suggested that the optimal endpoint for simulator training is the attainment of a predefined level (criterion-based training), rather than the completion of an arbitrary number of procedures, task repetitions, or hours using the simulator (time-based training) [9-11]. It can be doubted that all robot professionals completed their learning curve in basic robotic skills within $5 \mathrm{~h}$ of training. A multidisciplinary basic skills training and examination based on clear criteria can assure a proper end of training level for all trainees and can prevent that the learning curve of basic skills is completed on patients. 

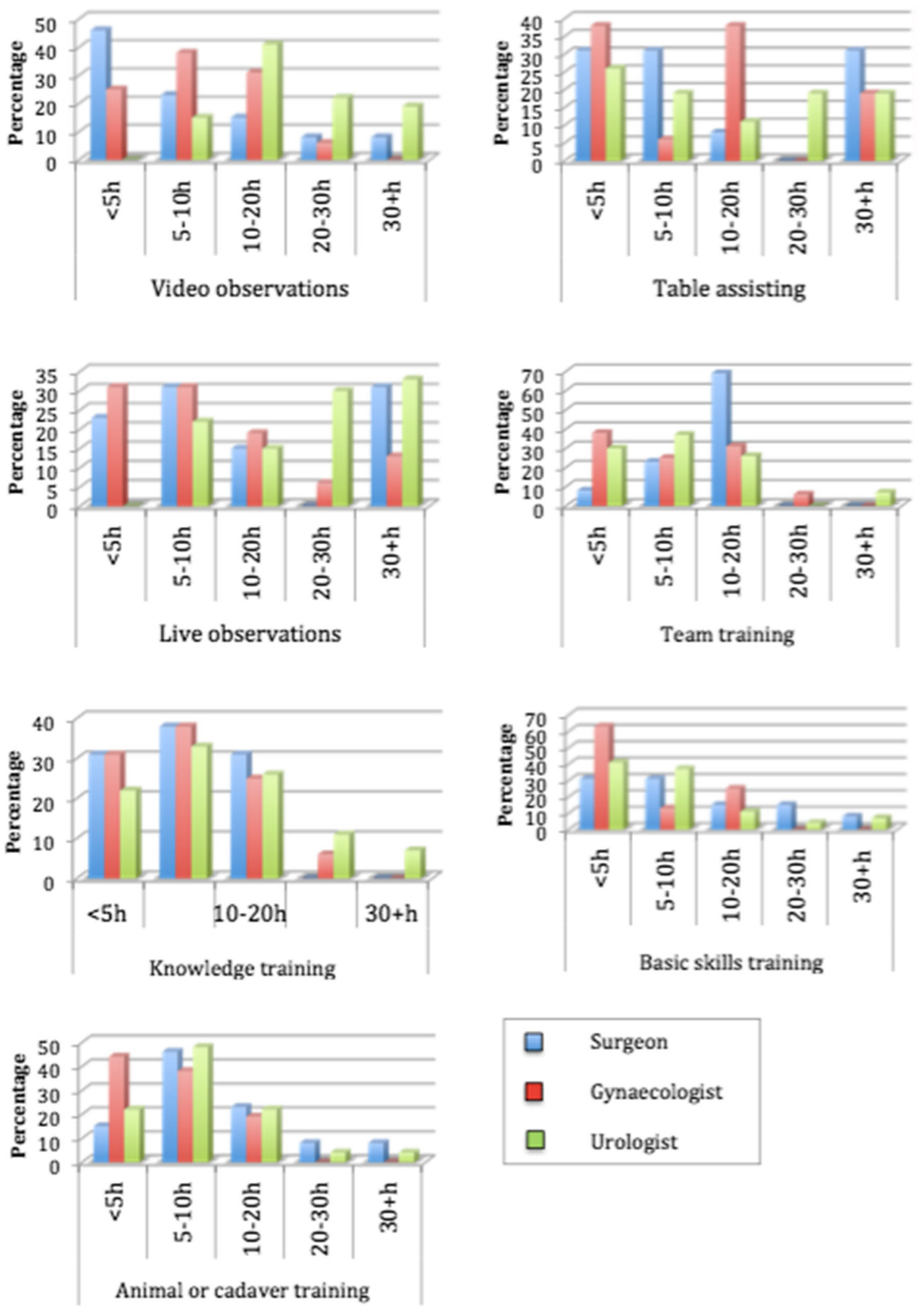

Fig. 3 Hours spent on different training types 


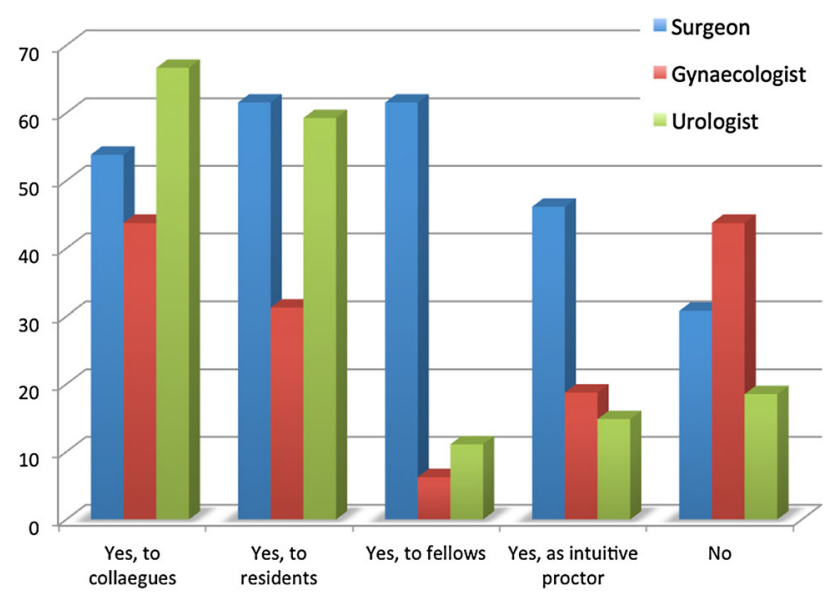

Fig. 4 Do you teach robot-assisted surgery?

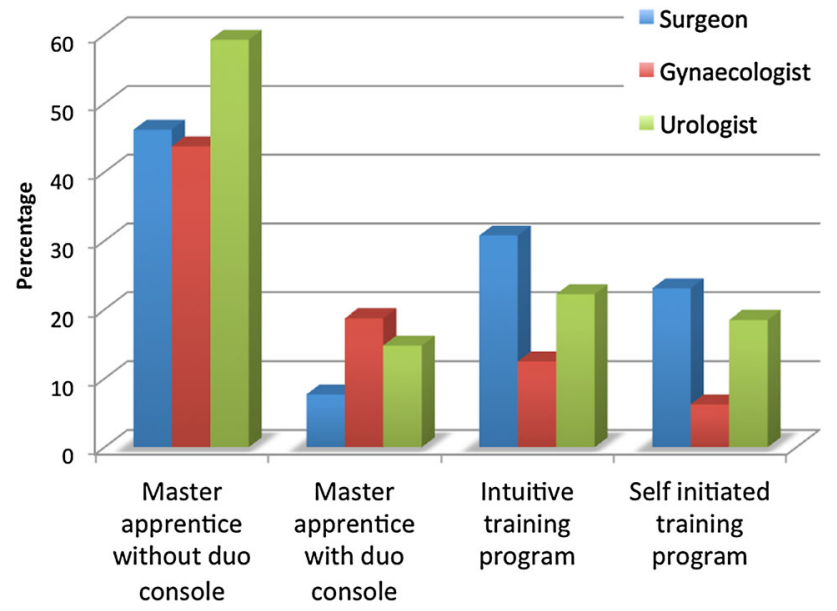

Fig. 5 How do you teach robot-assisted surgery?
Another finding is that almost all respondents had previous laparoscopic experience. Conventional laparoscopy was already quiet common in surgery, gynecology, and urology in The Netherlands. And most likely, those already interested in minimal invasive surgery started robot-assisted surgery. Therefore, most robot users had already previous experience in laparoscopy. New generations will most likely receive less laparoscopy training since the robot is taking a large share of minimal invasive procedures, especially in urology. This makes a basic training even more important since the principals of minimal invasive abdominal surgery, for instance working with the pneumoperitoneum, should be taught to a new generation.

We did not find any significant differences in robot training between the specialities. This is a positive finding and shows that all specialities put effort in basic training. This can be explained by the fact that the manufacturer offered all new robot costumers a mandatory introduction training to the da Vinci Surgical System, independent of their speciality. This training guaranteed a basic level for all robot users. Nevertheless, there is a wide range in training hours among all specialties since training after the introduction course was not mandatory and was self-initiated. For residents or fellows, it will be different since new users are not new customers, so the training program provided by the manufacturer is not mandatory for them.

As last research question, we asked the participants about their opinion on multidisciplinary basic skills training. The majority of the respondents favor an obliged training program and examination for the basics of robot skills training. As robotic surgery is expanding throughout various fields of surgery, there is still no consensus on a
Fig. 6 Do you agree on setting a structured and obliged training program for basic robotic skills? And do you think an examination or test should be installed before starting robotassisted surgery?

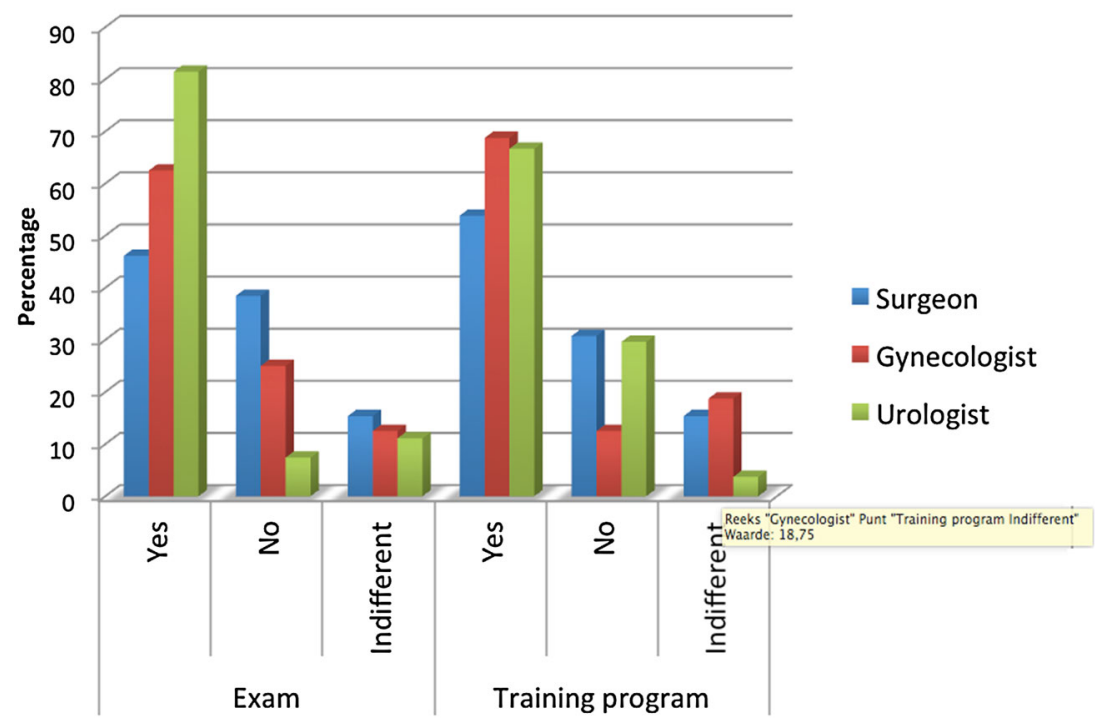


validated training curriculum. In 2010, the Dutch Health Care Inspectorate (IGZ) published a report 'Insufficiently prepared introduction of robotic surgery' [12]. This report noted insufficient criteria for the surgeon's competence before starting with robotic surgery in $50 \%$ of hospitals. Earlier, in 2007, a similar report was published about the risks of minimally invasive surgery that were being underestimated. Overall, these reports support the notion that training in endoscopic surgery needs to be improved and that a national multidisciplinary consensus is essential [13]. This confirms the increasing need for certified training and assessment criteria for residents, fellows, and surgeons. A structured multidisciplinary training program could be an effective starting point.

The majority of robot professionals in The Netherlands agree that a structured multidisciplinary training program should be implemented for the basics in robotic surgery. The time seems right since the majority of the professionals indicate that they teach residents robotic surgery and robotic skills acquirement is in particular improved at younger age. With the available literature on robot training $[14,15]$, a program can be developed with well-defined proficiency standards to safeguard the quality of care and prevent learning by doing directly on the patient.

A multidisciplinary training program could consist of items such as knowledge training, basic skills training, draping and docking, and patient positioning. Also, some general safety issues and anesthetic difficulties etcetera could be covered in a multidisciplinary training. In our opinion, these components could be trained and tested in no longer than 1- or 2-day course if participants come well prepared. After a broad criterion-based multidisciplinary basic training, a procedure and speciality-specific training could follow. Items that are more procedure specific such as video observations and table assisting are less suitable to cover multidisciplinary.

A limitation of our study is that the group studied was a selection (62\%) of all robot professionals. The participants were willing to complete a questionnaire about their training. Possibly, this led to a selection bias that influenced the reported opinions on training robotics. Another limitation is that we do not know the reason professionals would be against a training program. Before implementing a program, this would be very useful information to know.

\section{Conclusions}

Training of the current robot professionals is mostly dependent on local circumstances and the manufacturer of the robot system. Training is independent of the year of start with robotic surgery and speciality. To guarantee the quality of future training of residents and fellows in robot- assisted surgery, clear training goals should be formulated and implemented. Since this study shows that current training of different specialities does not differ, training in robotic surgery could be started by a multidisciplinary basic skills training and assessment.

Acknowledgments We thank all robotic professionals for their participation in this survey.

Disclosures Dr. Brinkman, Dr. de Angst, Dr. Schreuder, Dr. Schout, Dr. Jaarsma, Dr. Verweij, Dr. Hendrikx, and Dr. van der Poel have no conflict of interest or financial ties to disclose.

Open Access This article is distributed under the terms of the Creative Commons Attribution 4.0 International License (http://crea tivecommons.org/licenses/by/4.0/), which permits unrestricted use, distribution, and reproduction in any medium, provided you give appropriate credit to the original author(s) and the source, provide a link to the Creative Commons license, and indicate if changes were made.

\section{References}

1. Chang SL, Kibel AS, Brooks JD, Chung BI (2015) The impact of robotic surgery on the surgical management of prostate cancer in the USA. BJU Int 115:929-936. doi:10.1111/bju.12850

2. Liu H, Lawrie TA, Lu D, Song H, Wang L, Shi G (2014) Robotassisted surgery in gynaecology. Cochrane database Syst Rev 12:CD011422. doi:10.1002/14651858.CD011422

3. Antoniou SA, Antoniou GA, Koch OO, Pointner R, Granderath FA (2012) Robot-assisted laparoscopic surgery of the colon and rectum. Surg Endosc 26:1-11. doi:10.1007/s00464-011-1867-y

4. Abboudi H, Khan MS, Aboumarzouk O, Guru KA, Challacombe B, Dasgupta P, Ahmed K (2013) Current status of validation for robotic surgery simulators a systematic review. BJU Int. doi:10. 1111/j.1464-410X.2012.11270.x

5. Jarc AM, Curet M (2014) Construct validity of nine new inanimate exercises for robotic surgeon training using a standardized setup. Surg Endosc 28:648-656. doi:10.1007/s00464-013-3224-9

6. Su L-M (2010) Robot-assisted radical prostatectomy: advances since 2005. Curr Opin Urol 20:130-135. doi:10.1097/MOU. 0b013e328336257a

7. Brinkman WM, Schout BMA, Rietbergen JB, de Vries AH, van der Poel HG, Koldewijn EL, Witjes JA, van Merriënboer JJG (2014) Training robotic surgery in urology: experience and opinions of robot urologists. Int J Med Robot. doi:10.1002/rcs.1631

8. Wiener S, Haddock P, Shichman S, Dorin R (2015) Construction of a urologic robotic surgery training curriculum: how many simulator sessions are required for residents to achieve proficiency? J Endourol. doi:10.1089/end.2015.0392

9. Brinkman WM, Buzink SN, Alevizos L, De Hingh IHJT, Jakimowicz JJ (2012) Criterion-based laparoscopic training reduces total training time. Surg Endosc Other Interv Tech 26:1095-1101. doi:10.1007/s00464-011-2005-6

10. Gauger PG, Hauge LS, Andreatta PB, Hamstra SJ, Hillard ML, Arble EP, Kasten SJ, Mullan PB, Cederna PS, Minter RM (2010) Laparoscopic simulation training with proficiency targets improves practice and performance of novice surgeons. Am J Surg 199:72-80. doi:10.1016/j.amjsurg.2009.07.034

11. Ahlberg G, Enochsson L, Gallagher AG, Hedman L, Hogman C, McClusky DA, Ramel S, Smith CD, Arvidsson D (2007) Proficiency-based virtual reality training significantly reduces the 
error rate for residents during their first 10 laparoscopic cholecystectomies. Am J Surg 193:797-804. doi:10.1016/j.amjsurg. 2006.06.050

12. Dutch Health Care Inspectorate. www.igz.nl/zoeken/document. aspx?doc=Onvoldoende_zorgvuldigheid_bij_introductie_operatie robots\&URL=. Accessed 27 March 2011. No Title

13. Schreuder HWR, Wolswijk R, Zweemer RP, Schijven MP, Verheijen RHM (2012) Training and learning robotic surgery, time for a more structured approach: a systematic review. BJOG 119:137-149. doi:10.1111/j.1471-0528.2011.03139.x
14. Fisher RA, Dasgupta P, Mottrie A, Volpe A, Khan MS, Challacombe B, Ahmed K (2015) An over-view of robot assisted surgery curricula and the status of their validation. Int J Surg 13:115-123. doi:10.1016/j.ijsu.2014.11.033

15. Smith R, Patel V, Satava R (2014) Fundamentals of robotic surgery: a course of basic robotic surgery skills based upon a 14-society consensus template of outcomes measures and curriculum development. Int J Med Robot 10:379-384. doi:10.1002/ rcs. 1559 